\title{
RECENT RESULTS ON LEAD-ION ACCUMULATION IN LEAR FOR THE LHC
}

S. Baird, J. Bosser, J. Broere, C. Carli, M. Chanel, C. Hill, R. Ley, A. Lombardi, R. Maccaferri, S. Maury, D. Möhl, G. Molinari, H. Mulder, E. Tanke, G. Tranquille, F. Varenne and M. Vretenar

CERN, 1211 Geneva 23, Switzerland

I. Meshkov and E. Syresin

JINR, 141980 Dubna, Moscow Region, Russia

\begin{abstract}
To prepare dense bunches of lead ions for the LHC it has been proposed to accumulate the $4.2 \mathrm{MeV} / \mathrm{u}$ linac beam in a storage ring with electron cooling. A series of experiments is being performed in the low-energy ring LEAR to test this technique. First results were already reported at the Beam Crystallisation Workshop in Erice in November 1995. Two more recent runs to complement these investigations were concerned with: further study of the beam lifetime; the dependence of the cooling time on optical settings of the storage ring and on neutralization of the electron beam; tests in view of multiturn injection. New results obtained in these two runs in December 1995 and in April 1996 will be discussed in this contribution.
\end{abstract}

Presented at the "11th ICFA Beam Dynamics Workshop on Beam Cooling and Instability Damping' held on board a ship between Moscow and Nizhny Novgorod, 18-26 June 1996 


\section{INTRODUCTION}

After the completion of the antiproton programme, the Low-Energy Antiproton Ring (LEAR) becomes available to serve as an accumulator ring for heavy ions in the injector chain for the future Large Hadron Collider (LHC) [1]. The scheme proposed [2] for the injection of lead ions is sketched in Fig. 1. It is based on a 'present day' ECR source delivering a current of $100 \mu \mathrm{Ae}$ of $\mathrm{Pb}^{27+}$. Multiturn injection and accumulation with the aid of electron cooling are required to gain a factor of $\sim 125$ in intensity. A multiturn scheme has been proposed [3] which combines injection into the transverse and the longitudinal phase planes. This holds the promise of a large number of injectable turns, and facilitates the optimization of the resulting three-dimensional emittance distribution for electron cooling. With a linac cycling at $10 \mathrm{~Hz}$, cooling times faster than $100 \mathrm{~ms}$ are desirable to accumulate 20 pulses in $2 \mathrm{~s}$.

A series of machine experiments [4]-[7] has been performed to test the techniques required. Since the main duty of LEAR, up to the end of 1996, is to supply high-quality antiproton beams to its users, these tests were limited in time and scope. In fact multiturn injection and stacking could not be tried because they require a new configuration of bumpers and septa (Fig. 2) which is not compatible with the ultra slow extraction of LEAR.

A workshop report on the first two experimental sessions was given in Ref. [8]. In the present note we summarize the subsequent results (Dec. 1995 and April 1996 runs) which might be of interest to the community present at this symposium. 
Figure 1: Sketch of the lead-ion injection scheme for the LHC. 
Figure 2: Present configuration of LEAR and modifications proposed for future experiments. A thin septum and four fast bumper magnets have to be installed to test multiturn injection. The electron cooling is moved to straight section 2 to have different Twiss functions in the cooling and the injection section whilst keeping the symmetry of two of the lattice. It is also proposed to increase the cooling length in order to test the resulting improvement of the cooling strength.

\section{MACHINE PARAMETERS}

Experiments were performed with lead ions of three different charge states $(53+$ to $55+$ ) which can be selected with sufficient intensity after stripping the beam from the lead-ion linac 'linac 3' at $4.2 \mathrm{MeV} / \mathrm{u}$. Lead $55+$ was tested for the first time, the other charge states including $\mathrm{Pb}^{52+}$ had already been used in previous runs. Some comparative measurements were also made with protons from linac 2 (see Table 1). Seven different optical settings of LEAR ('machine 1-7', Table 2) were used to test the influence of the lattice functions on cooling and beam lifetime. To have sufficient margin in the choice of the dispersion $(D)$ and focusing $\left(\beta_{\mathrm{h}}\right)$ at the cooler, it was necessary to abandon the usual four-fold symmetry (same $D$ and $\beta$ in all four straight sections) of LEAR. However, twofold symmetry had to be retained resulting in identical Twiss parameters in the straight sections SS1 (injection) and SS3 (electron cooling) on the one hand, and in SS2 and SS4 on the other hand (Table 2). We plan to move the cooler in 1997 to SS2 (Fig. 2) so that the Twiss functions for injection and cooling can be adjusted more independently.

Care was taken to record the vacuum conditions at the beginning and at the end of each run and during intermediate times. Four mass spectrometers installed in differ- 
ent straight sections of LEAR were read via a PC to obtain the gas composition and to evaluate the expected lifetime. The average vacuum pressure and especially the gas composition turned out to be different from run to run (Table 3 ).

A pressure rise was observed, when large losses of ions occurred, especially when the injection kicker was not pulsed so that the whole beam was lost on the first turn. Pulsing the linac continuously with a repetition rate of $1 / 1.25 \mathrm{~s}$ whilst the LEAR kicker was disabled, the final increase in ring average pressure was as big as a factor of 2 (from typically $2 \times 10^{-11}$ to $4 \times 10^{-11}$ torr). The pressure rise was concentrated in those parts of the ring where the beam was lost.

Table 1: Main parameters of proton, lead-ion, and electron cooling beams

\begin{tabular}{|c|c|c|}
\hline & Protons & Ions $\left(\mathrm{Pb}^{53+}\right.$ and $\left.\mathrm{Pb}^{54+}\right)$ \\
\hline Ion energy & $50 \mathrm{MeV}$ & $4.2 \mathrm{MeV} / \mathrm{u}$ \\
\hline Velocity factor $\beta=v / c$ & 0.31 & 0.094 \\
\hline Current $I_{\mathrm{i}}$ & few $\mathrm{mA}$ & $20-25 \mu \mathrm{Ae}$ \\
\hline Corresponding no. of particles & few $10^{9}$ & $2-3 \times 10^{6}$ ions \\
\hline Electron cooling energy & $27 \mathrm{keV}$ & $2.3 \mathrm{keV}$ \\
\hline Cooling length/circumference $\eta_{\text {cool }}$ & 0.02 & 0.02 \\
\hline Electron beam radius $a_{\mathrm{e}}$ & $25 \mathrm{~mm}$ & $25 \mathrm{~mm}$ \\
\hline Magnetic field in cooler & $0.06 \mathrm{~T}$ & $0.06 \mathrm{~T}$ \\
\hline Typical electron current $I_{\mathrm{e}}$ & $1-2.2 \mathrm{~A}$ & $0.06-0.4 \mathrm{~A}$ \\
\hline
\end{tabular}

Table 2: Some data for the different optical settings of LEAR 'machines 1-7'. Lattice functions at the cooler are given by the values in SS1/SS3.

\begin{tabular}{|c|l|c|c|c|c|c|l|}
\hline Machine & \multicolumn{1}{|c|}{1} & \multicolumn{1}{c|}{2} & 3 & 4 & 5 & 6 & \multicolumn{1}{c|}{7} \\
\hline$\beta_{\mathrm{H}} \mathrm{SS} 1 / \mathrm{SS} 3[\mathrm{~m}]$ & 1.9 & 1.3 & 10.9 & 9.5 & 1.7 & 0.65 & 4.8 \\
$\beta_{\mathrm{v}} \mathrm{SS} 1 / \mathrm{SS} 3[\mathrm{~m}]$ & 6.4 & 9.6 & 4.1 & 10.5 & 13.4 & 5.5 & 5.0 \\
$D$ SS1/SS3 [m] & 3.5 & 0 & 0 & 0 & 9.8 & 0 & 5.0 \\
$\beta_{\mathrm{H}} \mathrm{SS} 2 / \mathrm{SS} 4[\mathrm{~m}]$ & 1.9 & 9.6 & 6.4 & 3.5 & 13.5 & 25.4 & 1.1 \\
$\beta_{\mathrm{v}} \mathrm{SS} 2 / \mathrm{SS} 4[\mathrm{~m}]$ & 6.4 & 11.9 & 14.8 & 7.2 & 14.9 & 5.3 & 6.2 \\
$D \mathrm{SS} 2 / \mathrm{SS} 4[\mathrm{~m}]$ & 3.5 & 9.8 & 9.6 & 9.9 & 0.06 & 10.1 & 2.0 \\
$Q_{\mathrm{H}}$ & 2.31 & 2.46 & 1.82 & 1.62 & 1.40 & 2.76 & 2.55 \\
$Q_{\mathrm{v}}$ & 2.62 & 2.42 & 2.42 & 2.42 & 2.24 & 2.72 & 2.70 \\
\hline
\end{tabular}


Table 3: Ring average vacuum conditions estimated from the readings of four gas analysers. No detailed analysis of the residual gas was done in the December 1994 run.

\begin{tabular}{|l|c|c|c|c|}
\hline \multirow{2}{*}{$\begin{array}{l}\text { Residual } \\
\text { gas }\end{array}$} & \multicolumn{4}{|c|}{$\begin{array}{c}\text { Partial pressures, in } 10^{-12} \\
\text { ring } \text { torr, }\end{array}$} \\
\cline { 2 - 5 } & Dec. 94 & June 95 & Dec. 95 & Apr. 96 \\
\hline $\mathrm{H}_{2}$ & - & 14.5 & 41.7 & 7 \\
$\mathrm{He}$ & - & 1.9 & 0.5 & 1.3 \\
$\mathrm{CH}_{4}$ & - & 1.4 & 3.4 & 2.7 \\
$\mathrm{H}_{2} \mathrm{O}$ & - & 1.4 & 0.9 & 0.8 \\
$\mathrm{CO}$ & - & 0.4 & 0.4 & 1.2 \\
$\mathrm{~N}_{2}$ & - & 0.1 & 0 & 3.4 \\
$\mathrm{O}_{2}$ & - & 0 & 0 & 0.5 \\
$\mathrm{Ar}$ & - & 0.1 & 0.6 & 0.3 \\
$\mathrm{CO}_{2}$ & - & 0.1 & 0.2 & 0.4 \\
\hline $\mathrm{Sum}$ & 11 & 19.9 & 47.7 & 17.6 \\
\hline
\end{tabular}




\section{BEAM LIFETIME MEASUREMENTS}

The beam lifetime $(\tau)$ was estimated by recording the ion intensity versus time deduced from the longitudinal Schottky signal, as in the previous runs [4]-[7]. The decay rate $1 / \tau=1 / \tau_{\text {vac }}+1 / \tau_{\mathrm{c}}$ has contributions due to the charge exchange with the residual gas and due to the presence of the cooling electron beam. The former is constant whereas the latter increases with electron current $\left(I_{\mathrm{e}}\right)$. From a plot of $1 / \tau$ vs. $I_{\mathrm{e}}$ we can deduce $1 / \tau_{\text {vac }}$ and $1 / \tau_{\mathrm{c}}\left(I_{\mathrm{e}}\right)$ (see the example reproduced in Fig. 3.)

We have used the formula of Franzke [9] to estimate $\tau_{\text {vac }}$ for the vacuum conditions given in Table 3 and found good agreement (Table 4) with the measured values. No difference in $\tau_{\text {vac }}$ between the different charge states $\mathrm{Pb}^{52+}$ to $\mathrm{Pb}^{55+}$ could be detected, in agreement with theory which predicts similar cross-sections.

In contrast to this a very strong and unexpected charge-state dependence of $1 / \tau_{\mathrm{c}}$ was found in the previous runs $[4,5]$. These observations were confirmed and complemented by the new results. The situation is summarized in Table 5 where we compile rate coefficients $\alpha_{\tau}$ (in units of $10^{-8} \mathrm{~cm}^{3} \mathrm{~s}^{-1}$ ). These give the slope of the $1 / \tau$ vs $n_{\text {eff }}$ curve where the electron beam density effective per turn is given by the true density of the electron beam and the fraction $\eta_{\text {cool }}$ of the circumference covered by the electron cooler $n_{\text {eff }}=n_{\mathrm{e}} \eta_{\text {cool }}$.

Figure 3: Dependence of the inverse lifetime on electron current for three different $\mathrm{Pb}$-ion charge states. The 'machine 4' optics is used in this example. 
Table 4: Beam lifetime due to charge exchange with the residual gas for the vacuum conditions summarized in Table 3. The 'theoretical values' are obtained by adding the decay rates due to the different components. The semi-empirical formula of Ref. [9] is used to obtain the electron capture - and loss cross-sections of the $\mathrm{Pb}$ ions with the molecules of the residual gas.

\begin{tabular}{|l|c|c|c|c|}
\hline Beam lifetime (s) & Dec. 94 & June 95 & Dec. 95 & Apr. 96 \\
\hline Theoretical & - & 17 & 13 & 12 \\
Measured & 20 & 16 & 10 & 9 \\
\hline
\end{tabular}

Table 5: Rate coefficients $\left(10^{-8} \mathrm{~cm}^{3} \mathrm{~s}^{-1}\right)$ for the different charge states and different lattice functions at the cooler

\begin{tabular}{|c|c|c|c|c|c|}
\hline \multirow[t]{2}{*}{ Run } & \multicolumn{4}{|c|}{$\begin{array}{l}\text { Rate coefficient } \\
\text { for the different } \\
\text { charge state }\end{array}$} & \multirow[t]{2}{*}{$\begin{array}{c}\text { 'Machine' } \\
\text { no. } \\
\text { (Table 2) }\end{array}$} \\
\hline & $\mathrm{Pb}^{52+}$ & $\mathrm{Pb}^{53+}$ & $\mathrm{Pb}^{54+}$ & $\mathrm{Pb}^{55+}$ & \\
\hline Dec. 94 & \multirow{6}{*}{11} & 64 & & \multirow{6}{*}{12} & 1 \\
\hline June 95 & & 60 & 9 & & 1 \\
\hline Dec. 95 & & 63 & 5 & & 4 \\
\hline \multirow[t]{3}{*}{ Apr. 96} & & 60 & 9 & & 1 \\
\hline & & 60 & 6 & & 4 \\
\hline & & & 7 & & 7 \\
\hline
\end{tabular}

For the LEAR cooler (Table 1 ) at $2.3 \mathrm{keV}$ electron energy:

$$
n_{\mathrm{eff}} \simeq 2 \times 10^{-2} n_{\mathrm{e}}=2.2 \times 10^{6}\left[\mathrm{~cm}^{-3} \mathrm{~A}^{-1}\right] \times I_{\mathrm{e}} .
$$

The following can be observed from Table 5: the rate coefficient of $\mathrm{Pb}^{53+}$ is unusually strong. Values close to $60 \times 10^{-8} \mathrm{~cm}^{3} \mathrm{~s}^{-1}$ were measured in all runs independent of the optical setting (machine 1 and 4) of LEAR. For $\mathrm{Pb}^{52+}$ and $\mathrm{Pb}^{55+}$, coefficients of the order of $10 \times 10^{-8} \mathrm{~cm}^{3} \mathrm{~s}^{-1}$ were found. Both were measured only once, for $\mathrm{Pb}^{52+}$ with the normal LEAR lattice and $\mathrm{Pb}^{55+}$ with 'machine 4' which has large beta-functions and zero dispersion at the cooler. For $\mathrm{Pb}^{54+}$ coefficients in the range of $5-9 \times 10^{-8} \mathrm{~cm}^{3} \mathrm{~s}^{-1}$ were measured, apparently depending on the lattice functions at the cooler.

We note that these rate coefficients are not easily explainable by existing theory. For radiative electron capture, coefficients of only $2-3 \times 10^{-8}$ are expected at the electron temperatures of the LEAR cooler which are estimated to be 0.1 to $0.2 \mathrm{eV}$ from the $H^{0}$ rate when protons are cooled. Rates up to 10 times faster than radiative recombination have been reported in the literature for special partially stripped ions and attempts to explain them by resonant dielectronic capture have been made, see Ref. [5] and references given therein. The same explanation could apply to $\mathrm{Pb}^{52+}, \mathrm{Pb}^{54+}$, and $\mathrm{Pb}^{55+}$. However, the rate of $\mathrm{Pb}^{53+}$ is especially fast and points to an unusually strong capture resonance or other mechanisms.

Several tests were made to further elucidate this question. The longitudinal magnetic field of the cooler was varied between 200 and $800 \mathrm{G}$ for $\mathrm{Pb}^{53+}$ and $\mathrm{Pb}^{54+}$. No marked differences in the lifetimes were detected. The energy of the electron beam was set off by a large amount. In this case the lifetime increased and reached a value only slightly shorter than the 'vacuum lifetime' in the absence of the electron beam. The difference 
can be explained by the pressure bump created by the collector inefficiency. When the electron beam was neutralized a further reduction of the 'vacuum lifetime' was observed which was especially pronounced when neutralization jumps occurred. These effects were the same for all the charge states investigated.

For the April 1996 run a movable scintillation screen was installed in the first bending magnet downstream of the cooling section to intercept the $\mathrm{Pb}^{(Q-1)+}$ ions created due to electron capture by circulating $\mathrm{Pb}^{Q+}$ ions. A strong counting rate, especially for circulating $\mathrm{Pb}^{53+}$, was observed when the scintillator covered the expected position of the recombined ions (in our case $\sim 25 \mathrm{~mm}$ horizontally outwards from the circulating beam). A large reduction of the signal occurred when the electron cooling was switched off or when the screen was withdrawn by more than $25 \mathrm{~mm}$ from the main beam thus indicating that indeed capture of cooling electrons by cooled ions is responsible for at least a fraction of the losses. More refined experiments are in preparation.

To summarize the results of this section: we have found that both charge exchange with the residual gas (including the neutralizing ions in the cooling section) and capture of cooling electrons have a very critical influence on the lifetime of the circulating ion beam. A high-quality vacuum (pressure and gas composition) is essential to obtain the desired $\tau_{\mathrm{vac}} \approx 40 \mathrm{~s}$. Losses — both of the circulating beam and of the electron beam have to be minimized to avoid outgassing. Full neutralization of a strong cooling beam is problematic.

The recombination of $\mathrm{Pb}^{53+}$ leads to an uncomfortably short lifetime $\left(\tau_{\mathrm{c}} \approx 2 \mathrm{~s}\right.$ at $\left.I_{\mathrm{e}}=400 \mathrm{~mA}\right)$. For $\mathrm{Pb}^{54+}$ the lifetime $\left(\tau_{\mathrm{c}} \approx 16\right.$ to $20 \mathrm{~s}$ at $\left.I_{\mathrm{e}}=400 \mathrm{~mA}\right)$ is acceptable.

\section{MEASUREMENTS OF THE COOLING TIME}

The measurements of the cooling time had two main objectives:

i) to test the effect of neutralization of the electron beam

ii) to assess the influence of the lattice functions of the storage ring.

As in the previous runs the evolution of the momentum spread was deduced from the Schottky noise signal near a harmonic $(n)$ of the revolution frequency. The transverse emittances were observed via the Schottky signal induced by the coasting beam at one of the betatron sideband frequencies $(n \pm Q) f_{\text {rev }}$ on a horizontal or vertical position pick-up. A second method to observe the transverse emittance is based on Beam Ionization Profile Monitors (BIPMs) which use position-sensitive channel plates to detect the ionization of the residual gas and thereby the beam width and height.

Two different techniques to measure the 'cooling times' were used: The 'cooling down' of the injected beam was analysed and/or a well-cooled beam was kicked (using a fast 'ejection' kicker to provide a deflection smaller than the aperture) and the decay of the betatron oscillation was recorded with the BIPM (the Schottky noise is more difficult to use in this situation).

The beam after injection had 'typical' emittances of $\Delta p / p=1 \times 10^{-3}, 2 \mathrm{rms}$; $\epsilon_{\mathrm{v}}=7 \pi \mathrm{mm} \mathrm{mrad}, \epsilon_{\mathrm{H}}=50 \pi \mathrm{mm}$ mrad. Equilibrium values, e.g. for cooling with $I_{\mathrm{e}}=350 \mathrm{~mA}$ are $\Delta p / p \approx 0.15 \times 10^{-3}, \epsilon_{\mathrm{v}} \approx \epsilon_{\mathrm{H}} \approx 4 \pi \mathrm{mm}$ mrad. For the comparison of different lattices and neutralization states we use the 'cooling down times' (hereafter simply called 'cooling times') defined as:

$\tau_{\Delta p}:$ the time it takes to decrease $\Delta p / p$ from 1 to $0.2 \times 10^{-3}$;

$\tau_{\mathrm{H}}$ : the time for $\epsilon_{\mathrm{H}}$ to decrease from the initial value down to $4 \pi \mathrm{mm} \mathrm{mrad}$.

Clearly, for the injected beam, cooling proceeds simultaneously in all three planes whereas with the kick method the cooling of horizontal betatron oscillations can be tested 
for a beam that is already cooled in the longitudinal and vertical degree of freedom. Thus the kick method simulates to some extent the conditions after horizontal multiturn injection.

The December 1995 run was mainly used to test the influence of neutralization. Owing to the improvements made $[6,8]$ it had become possible to work with stable neutralization levels that could be chosen between 0 and $100 \%$ for currents up to $I_{\mathrm{e}}=200 \mathrm{~mA}$. A large number of measurements were made varying the neutralization and/or the intensity of the electron beam. The injected beam method was used for the 'machine 4' optics and the kick method for 'machine 1' and 'machine 4'. Results may be summarized by stating that no appreciable gain in the cooling times was obtained with neutralization. In some situations we saw a small improvement whereas in other cases neutralization was detrimental to the cooling process. More experiments are necessary to get a clearer picture.

The influence of the lattice functions was tested in the April run where care was taken to have zero neutralization during all measurements. A first series of tests was performed with protons which are easier to handle due to the long lifetime and the higher intensity. The 'kicked-beam method' was applied simulating a beam with a horizontal emittance of about $40 \pi \mathrm{mm}$ mrad in all cases. The electron current was $1.2 \mathrm{~A}$. Results of $\tau_{\mathrm{H}}$ for four different optical settings are plotted in Fig. 4 against the horizontal focusing function $\left(\beta_{\mathrm{H}}\right)$ at the cooler. It appears that 'machine 1' $\left(\beta_{\mathrm{H}} \approx 2 \mathrm{~m}\right)$ and 'machine 7 ' $\left(\beta_{\mathrm{H}} \approx 5 \mathrm{~m}\right)$ are fairly close to the optimum. It should be mentioned that the 'machines' considered, in addition to the difference in $\beta_{\mathrm{H}}$ also have different $D$ at the cooler. Therefore the effect of $D$ may be superimposed on the influence of $\beta_{\mathrm{H}}$, and may even be the dominant effect. 
Figure 4: Plot of the proton beam cooling time versus the horizontal $\beta$-function at the cooler. The electron current was $I_{\mathrm{e}}=1.2 \mathrm{~A}$. The time to cool $\sim 2 \times 10^{9}$ protons at $50 \mathrm{MeV}$ after a kick corresponding to $40 \pi \mathrm{mm}$ mrad to $4 \pi \mathrm{mm}$ mrad horizontal emittance is given. The measured points are for 'machines $6,1,7$ and 4 ' $\left(\beta_{\mathrm{H}}=0.65,1.9,4.8\right.$ and $\left.9.5 \mathrm{~m}\right)$, see Table 2 . The curve corresponds to a fit $\tau_{\mathrm{H}}=0.2 \beta_{\mathrm{H}}^{2}+13 / \beta_{\mathrm{H}}^{3 / 2}$. Note that in addition to $\beta_{\mathrm{H}}$, the dispersion $D$ is different for the 'machines' tested. This might add to the effect of $\beta_{\mathrm{H}}$ and could even be the dominant factor for the difference of cooling time.

Guided by the results for protons (which are for different electron energy and current!) three different optical settings were explored with $\mathrm{Pb}^{54+}$ ions. The measurements are illustrated in Fig. 5 in the form of a plot of the cooling rate $\left(1 / \tau_{\mathrm{H}}\right)$ vs. electron current $\left(I_{\mathrm{e}}\right)$. One notes that 'machine 7 ' has now the fastest horizontal cooling, 'machine 1 ' leads to a somewhat longer one and 'machine 4' to a significantly longer cooling time $\tau_{\mathrm{H}}$. Qualitatively these observations can be explained by the interplay of the ion velocity spread $\Delta v_{\mathrm{H}}=\beta c \sqrt{\epsilon_{\mathrm{H}} / \beta_{\mathrm{H}}}$ which tends to reduce the cooling force when $\beta_{\mathrm{H}}$ becomes too small, and the 'overlap factor' $a_{\mathrm{H}} / a_{\mathrm{e}}$ which reduces the cooling efficiency when the ion beam radius $a_{\mathrm{H}}=\sqrt{\epsilon_{\mathrm{H}} \beta_{\mathrm{H}}}$ becomes larger than the electron beam $\left(a_{\mathrm{e}}=25 \mathrm{~mm}\right.$ in our case). Naïvely one would expect an optimum for $a_{\mathrm{H}} \approx a_{\mathrm{e}}$ which for $\epsilon_{\mathrm{H}}=40 \pi \mathrm{mm} \mathrm{mrad}$ gives an 'optimum' $\beta_{\mathrm{H}} \approx 15 \mathrm{~m}$ compared with the measured $\beta_{\mathrm{H}}=5 \mathrm{~m}$. An 'effective' electron beam radius $a_{\mathrm{eff}} \approx 15 \mathrm{~mm}$ (instead of $a_{\mathrm{e}}=25 \mathrm{~mm}$ expected from the design of the electron gun) could explain the measurements. 
Figure 5: Cooling rate (inverse of time to go from $40 \pi$ to $4 \pi \mathrm{mm} \operatorname{mrad}$ horizontally) as a function of the electron current, for $\sim 10^{7} \mathrm{~Pb}^{54+}$ ions at $4.2 \mathrm{MeV} / \mathrm{u}$.

To summarize the results of this section we have found (so far?) no advantage in using a strongly neutralized electron beam. However, the possibility to monitor and control neutralization at a constant level (especially $\eta=0$ ) has proven essential. A strong dependence of the (horizontal) cooling time on the lattice functions has been observed with an optimum $\left(\beta_{\mathrm{H}} \approx 5 \mathrm{~m}\right)$ lower than expected $\left(\beta_{\mathrm{H}} \approx 10-15 \mathrm{~m}\right)$ from simple theory. The influence of the dispersion $(D)$ in the cooling section, which could influence these results, has to be investigated in future runs.

\section{TESTS TO PREPARE FOR MULTITURN INJECTION}

For the multiturn scheme which combines injection into horizontal and longitudinal phase space [3] the energy of the linac has to be ramped by about $12 \times 10^{-3}$ in $50 \mu \mathrm{s}$ (about 20 turns in LEAR). The transfer line must have sufficient acceptance to transmit this momentum width $\left(\delta \Delta / p=6 \times 10^{-3}\right)$ plus the instantaneous momentum spread $\left(\Delta p / p \approx 2 \times 10^{-3}, 4 \mathrm{rms}\right)$ of the linac beam. At the injection septum the dispersion of the line must be $|D|<0.2 \mathrm{~m}$ to have a beam displacement $d \lesssim 1 \mathrm{~mm}$ during the ramp. A new optics of the $\sim 100 \mathrm{~m}$ long transfer line including the 'U-turn' between linac 3 and LEAR has been calculated [10]. To test this optics $\mathrm{Pb}^{53+}$ and $\mathrm{Pb}^{54+}$ were simultaneously transmitted by adjusting the magnetic filter line after the stripper to a large momentum width. After fine adjustment, the two beams passed the whole line, and the beam spots on a scintillator screen in front of the LEAR septum were superimposed with good accuracy. 
This proves that the momentum acceptance of the line was greater than $18 \times 10^{-3}$ and the residual dispersion at the septum was small, as required.

A second series of tests concerned the energy ramping of the linac. An energy change of $70 \mathrm{keV}$ (corresponding to a momentum shift $\delta p / p \approx 8 \times 10^{-3}$ ) in a time $\Delta t \gtrsim 60 \mu \mathrm{s}$ could be obtained by modulating the RF level of tank 3 and simultaneously the phase of the debuncher cavity installed in the line downstream of the linac [6, 11]. As mentioned, only single-turn $\left(t_{\mathrm{rev}} \approx 2.7 \mu \mathrm{s}\right)$ injection into LEAR was possible. However, the effect of the energy variation could be tested by displacing the timing of the injection kicker 'statically' along the ramp.

From the longitudinal Schottky scan in LEAR the mean energy and the energy spread as a function of the injection timing could be deduced. After careful synchronization of tank 3 and the debuncher, the momentum spread of the single-turn injected beam $\left(\Delta p / p \approx 2 \times 10^{-3}, 4 \mathrm{rms}\right)$ was virtually independent of the injection timing all along the ramp and practically the same as with constant linac energy.

These results look promising for multiturn injection with energy ramping.

\section{CONCLUSIONS}

Charge exchange with the residual gas and recombination with cooling electrons limit the lifetime of the partially stripped ion beam.

Residual gas pressures of $\leq 5 \times 10^{-12}$ torr for $\mathrm{H}_{2}$ and $<1 \times 10^{-12}$ torr for heavier residual gas components like $\mathrm{N}_{2}$ are desirable to obtain a good 'vacuum lifetime'. Recombination, especially for $\mathrm{Pb}^{53+}$, is stronger than expected. The lifetime of $\mathrm{Pb}^{54+}$ at the nominal electron current of $400 \mathrm{~mA}$ is acceptable for 2 to 4 seconds of storage. Optimum beta-functions at the electron cooler are found to be $\sim 5 \mathrm{~m}$ instead of the 10 to $15 \mathrm{~m}$ expected from simple theory.

The required fast cooling times have been obtained, but only at low intensity so far. A factor of more than 100 in intensity has to be gained by multiturn injection and stacking. At this high intensity collective effects, which have not shown up in the experiments so far, can become important.

\section{References}

[1] The LHC Study Group (eds. P. Lefèvre and T. Pettersson), The Large Hadron Collider, Conceptual Design, CERN/AC/95-05 (LHC) (CERN, Geneva, 1995).

[2] P. Lefèvre and D. Möhl, A Low Energy Accumulator Ring of Ions for LHC, report CERN/PS/93-62 (DI), 1993, and Proc. Workshop on Beam Cooling, Montreux, 1993, J. Bosser (ed.), CERN 94-03, p. 411.

[3] S. Maury and D. Möhl, Combined Longitudinal and Transverse Multiturn Injection in a Heavy Ion Accumulator Ring, internal report PS/AR/Note 94-12, 1994.

[4] S. Baird, J. Bosser, M. Chanel, J. Duran, R. Giannini, P. Lefèvre, R. Ley, R. Maccaferri, S. Maury, I. Meshkov, D. Möhl, G. Molinari, F. Motsch, H. Mulder, U. Oeftiger, J.C. Perrier, E. Roux, G. Tranquille and F. Varenne, First Electron Cooling Test with $\mathrm{Pb}^{53+}$ Ions (Performed in December 1994), internal report PS/AR/Note 95-06 (MD), 1995.

[5] S. Baird, J. Bosser, C. Carli, M. Chanel, P. Lefèvre, R. Ley, R. Maccaferri, S. Maury, I. Meshkov, D. Möhl, G. Molinari, F. Motsch, H. Mulder, G. Tranquille and F. Varenne, Beam Lifetime Measurements for $\mathrm{Pb}^{52+}, \mathrm{Pb}^{53+}$ and $\mathrm{Pb}^{54+}$ Ions Subject to Electron Cooling in LEAR (Performed in June 1995), internal report PS/AR/Note 95-12, 1995 and Phys. Lett. B361 (1995) 184. 
[6] S. Baird, J. Bosser, C. Carli, M. Chanel, R. Ley, R. Maccaferri, S. Maury, I. Meshkov, D. Möhl, G. Molinari, E. Syresin, G. Tranquille and F. Varenne, Injection, Storage and Cooling Tests of $\mathrm{Pb}^{53+}, \mathrm{Pb}^{54+}$, and $\mathrm{Pb}^{55+}$ Ions in LEAR (MD performed in December 1995), internal report PS/AR/Note 96-08 (MD), 1996.

[7] J. Bosser, J. Broere, C. Carli, M. Chanel, C. Hill, R. Ley, A. Lombardi, R. Maccaferri, S. Maury, D. Möhl, G. Molinari, H. Mulder, E. Tanke and G. Tranquille, LEAR Machine Studies to Test Ion Accumulation for the LHC. Summary, March-April 1996, internal report PS/AR/Note 96-09 (MD), 1996.

[8] J. Bosser, Review of Recent Work on Electron Cooling at LEAR, Proc. Workshop on Crystalline Beams and Related Issues, Erice, 1995, J. Hangst and A.G. Ruggiero (eds.), to be published, preprint CERN/PS/96-13 (AR).

[9] B. Franzke, Vacuum Requirements for Heavy Ion Synchrotrons, Proc. Part. Acc. Conf., IEEE Trans. Nucl. Sci. NS-28 (1981) 2116.

[10] C. Carli, A New Optics of the Linac 3-LEAR Transfer Line for Multiturn Injection, CERN internal report, to be published

[11] J. Broere, A. Lombardi, E. Tanke and M. Vretenar, Static and Dynamic Ramping of the Energy of the Beam from Linac 3, CERN internal report, to be published. 